\title{
Prognostic significance of peritoneal lavage cytology in gastric cancer in Singapore
}

\author{
Esther W.L. Chuwa ${ }^{1}$, Lay-Wai Khin ${ }^{2}$, Weng-Hoong Chan ${ }^{1}$, Hock-Soo Ong ${ }^{1}$, and WaI-Keong Wong ${ }^{1}$ \\ ${ }^{1}$ Department of Surgery, Singapore General Hospital, Outram Road, Singapore 169608 \\ ${ }^{2}$ Clinical Trials and Epidemiology Research Unit, Singapore
}

\begin{abstract}
Background. Peritoneal lavage cytology has been included as part of the staging process in the 13th edition of the Japanese Classification of Gastric Cancer. However, this procedure has neither been studied nor established in our population. We aimed to evaluate its prognostic relevance among our patients with gastric cancer.

Methods. A total of 142 consecutive patients with gastric carcinoma were recruited prospectively. All had histologically proven gastric carcinomas and had undergone laparotomy and intraoperative peritoneal lavage for cytological examination at Singapore General Hospital. The fluid recovered was centrifuged and stained by the Papanicolau method. All patients were followed up with endpoints of cancer recurrence and mortality.

Results. There were 91 men and 51 women; 36 patients $(25.4 \%)$ had positive peritoneal lavage. Patients with advanced macroscopic features, presence of vascular invasion, nodal involvement, advanced depth of tumor invasion and metastatic disease tended to have positive lavage, by univariate logistic regression analysis. Despite curative resections, patients with positive cytology had a more dismal disease-free survival (mean, 27 months vs 53 months; $P<$ 0.0001 by log rank test) and higher recurrence rate $(54.5 \%$ vs $19.3 \% ; P=0.007$ by $\log$ rank test). There was also a trend towards earlier recurrences (median, 8 months vs 11 months; $\boldsymbol{P}=\mathbf{0 . 3 7}$ ). By multivariate Cox regression stepwise analysis, advanced depth of tumor invasion and positive lavage cytology were found to be independent poor prognostic factors for disease-free survival.

Conclusion. Positive peritoneal lavage cytology correlated well with advanced features of gastric cancer. It is an independent poor prognostic factor and the procedure should be routinely performed. Integration of lavage status into our current staging systems may serve as a guide for adjuvant therapeutic options to improve the survival of gastric cancer in our population.
\end{abstract}

Offprint requests to: E.W.L. Chuwa

Received: February 7, 2005 / Accepted: July 7, 2005
Key word Peritoneal lavage cytology $\cdot$ Gastric cancer

\section{Introduction}

Peritoneal carcinomatosis represents one of the most common causes of failure after "curative" surgery for gastric cancer. This is believed to be due to the presence of malignant cells that have already seeded at the time of operation but cannot be detected using conventional diagnostic tools. Cytological examination using peritoneal washes obtained at the time of surgery has been used for assessing the presence of free cancer cells in the peritoneal cavity $[1,2]$. Data obtained by cytological examination of peritoneal washes have been included as one of the prognostic factors in the staging of gastric cancer by the Japanese Research Society for Gastric Cancer [3]. Recently, there has been a move towards combined-modality therapy to improve the outcome of advanced gastric cancer. Integration of lavage status into our current staging systems not only allows disease prognostication but also serves as a guide to adjuvant therapeutic options such as preoperative chemoirradiation [4] or intraperitoneal chemotherapy [5]. However, the sensitivity of peritoneal lavage cytology has been reported to be relatively low, ranging from $22 \%$ to $30 \%$ in gastric carcinoma involving the serosa [6-8]. As up to $50 \%$ of patients with serosal invasion are known to develop peritoneal recurrence even when curative resections are performed [8], several investigators have recognized the need for more sensitive molecular methods of detecting peritoneal metastasis compared with conventional cytodiagnostic methods. In this article, we report our study of the prognostic relevance of free cancer cells in the peritoneal washings among our population with gastric cancer and its potential impact on our current management strategy. 


\section{Patients and methods}

Between March 1998 and March 2002, a total of 142 consecutive patients receiving treatment from the upper gastrointestinal team at the Department of General Surgery, Singapore General Hospital, were prospectively recruited. All had histologically proven gastric carcinoma from endoscopic gastric biopsies and had undergone laparotomy and intraoperative peritoneal lavage for cytological examination. These patients had fulfilled the following criteria: (1) no preoperative chemotherapy; (2) no other primary malignancy; (3) no previous abdominal operations resulting in dense adhesions that would preclude accurate sampling; and (4) a peritoneal lavage sample which was sufficient for definitive diagnosis.

Upon entering the abdominal cavity prior to tumor handling, $200 \mathrm{ml}$ of warm normal saline was introduced and manually dispersed. At least $20 \mathrm{ml}$ of fluid was subsequently recovered from several regions of the abdominal cavity, including the pelvis and paracolic gutters. The fluid was then centrifuged for $5 \mathrm{~min}$ at $1500 \mathrm{rpm}$. The nucleated cell layer was smeared onto a glass slide and stained by the Papanicolau method. Cytological examination was performed by experienced cytopathologists. Cytological findings were graded from class I to V according to Papanicolau's classification, with classes IV and V defined as positive [9]. Standard D2 lymphadenectomy, according to the criteria described by the Japanese Research Society for Gastric Cancer [3], was routinely performed for all resections intended for cure. Resections were deemed curative when no gross residual disease was evident at the time of the operation, with tumor-free resection margins on histological examination. Resected specimens were examined histopathologically, with depth of invasion (pT) and the number of nodal metastases $(\mathrm{pN})$ evaluated and staged according to the 1997 International Union Against Cancer (UICC)/American Joint Committee on Cancer (AJCC) TNM classification (fifth edition).

All patients were followed up for at least 1 year or until death. Postoperative evaluation included interim history, physical examination, and hematological and blood biochemistry panels. Check upper endoscopy was performed 6- to 12-monthly. Computerized tomography of the abdomen, bone scintigraphy, and chest $\mathrm{X}$-ray were performed whenever clinical symptoms, physical findings, or investigations implied recurrence. Diagnoses of disease recurrence were made on the basis of radiological or endoscopic findings. Peritoneal recurrence was determined clinically, based on symptoms or physical examination of bowel obstruction and ascites. Cytological confirmation was obtained only for patients who underwent paracentesis or relaparotomy. Disease-free survival was defined as the time from the date of the primary treatment to the date of first recurrence. Overall survival was defined as the time from the date of the primary treatment to the date of death.

Prognostic analyses were performed with cancerrelated death and disease recurrence as primary endpoints. Statistical comparisons were made using the $\chi^{2}$ test and Fisher's exact test as appropriate. The sensitivity, specificity, and predictive value of peritoneal lavage cytological examination were calculated using Confidence Interval Analysis (CIA) software for clinical studies version 2.1.0 (Trevor N Bryant, Bristol, UK). Clinicopathological factors associated with positive cytological examination were analyzed by univariate and multivariate logistic regression. To determine which variables were independent prognostic factors for overall survival and disease-free survival, Cox regression analysis, in a backward stepwise procedure, was applied. We used $P<0.1$ as the cutoff value for statistical significance for variable selection for the multivariate modeling in order not to miss any potentially important predictors. Statistical significance remained conventionally defined as $P<0.05$ in the univariate and multivariate models. Survival rates were obtained by the Kaplan-Meier method and compared by log rank test. Statistical calculations were performed using the SPSS (Chicago, IL, USA) version 11.5 advanced statistical software module.

\section{Results}

A total of 142 patients were studied. There were 91 men and 51 women (male-to-female ratio, 1.78:1). The median age was 67.2 years (range, $24.0-86.1$ years). There was no operative-related mortality, and the overall morbidity was $17.6 \%(25 / 142)$. Common complications included chest infection $(n=11)$ and wound infection $(n=5)$. The median follow-up time was 11.8 months for all patients (range, 2 to 59 months) and 36 months (range, 13 to 59 months) for surviving patients. Two patients were lost to follow-up. Two patients died of other causes (one, stroke and the other, acute cardiovascular event). These four patients were negative for free peritoneal malignant cells and were excluded from the survival analysis. At the last follow-up, 52 patients $(36.6 \%)$ were dead of disease.

In total, 36 patients $(25.4 \%)$ had positive peritoneal malignant cytology. The patients' demographic, clinical, and tumor characteristics are listed in Table 1. The patients in both groups were comparable in distribution of age, sex, ethnic group, and presence of comorbidities. There was a significant association between the tumor stage, depth of tumor invasion, nodal involvement, intent of operation, disease recurrence, and overall mor- 
Table 1. Patients' demographic and clinical features and tumor characteristics

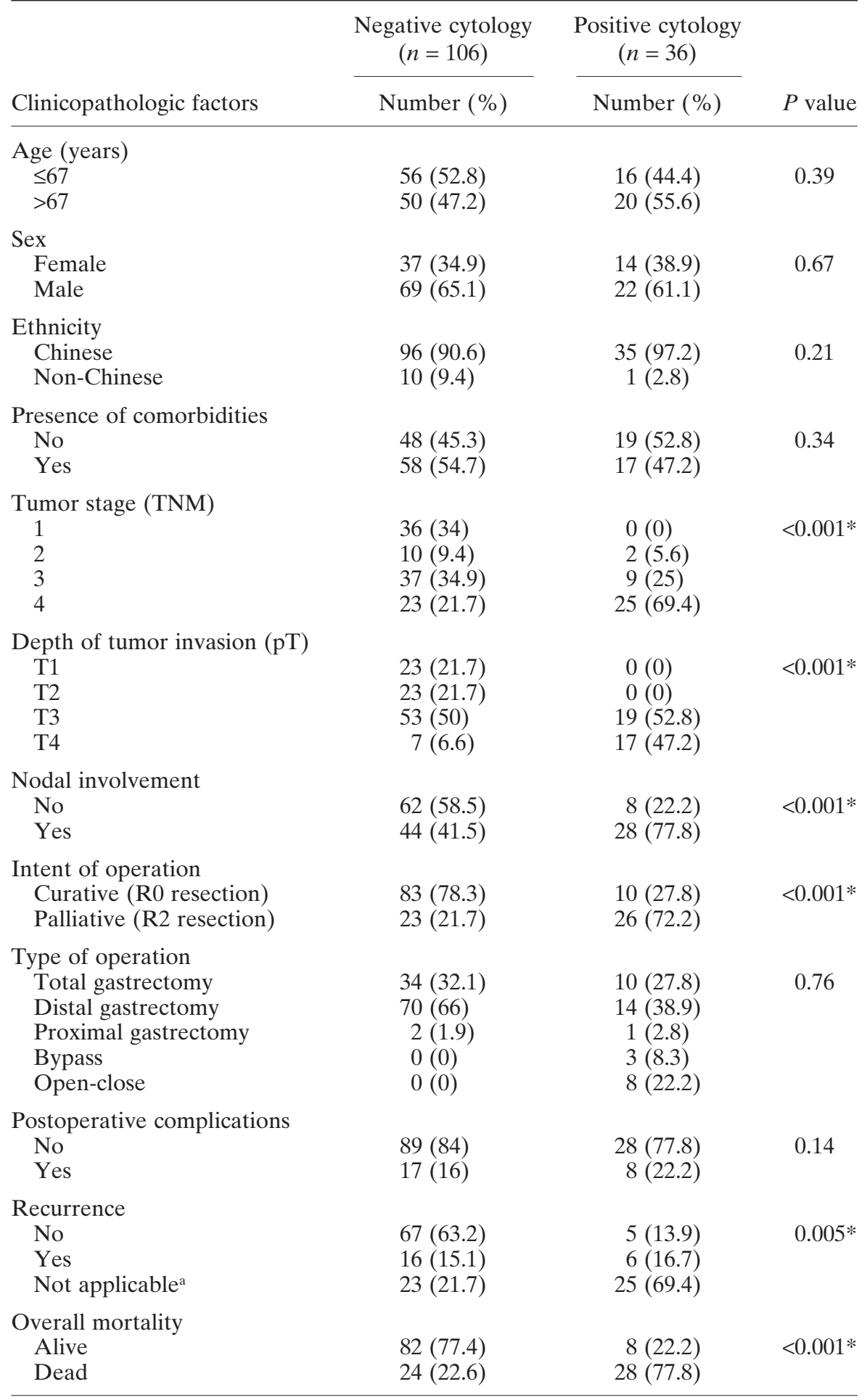

* Significant

${ }^{a}$ Due to presence of residual tumor or distant metastasis at the time of surgery

tality with peritoneal cytology positivity. For patients with positive peritoneal cytology, all had tumor invasion of the serosal surface. Peritoneal dissemination was grossly evident in 17 patients and was confirmed histopathologically. Excluding tumors with serosal involve- ment alone (T3), the sensitivity of peritoneal lavage cytology in detecting peritoneal disease was $70.8 \%$ (95\% confidence interval, 50.8-85.1) and specificity was $70.8 \%$ (95\% confidence interval, 58.8-80.4). The positive predictive value of peritoneal lavage cytology was 
Table 2. Factors influencing the positivity of peritoneal cytological examination, using univariate and multivariate logistic regression, among patients with serosa-positive (pT3, pT4) tumors $(n=96)$

\begin{tabular}{|c|c|c|c|c|c|c|}
\hline \multirow{2}{*}{$\begin{array}{l}\text { Clinicopathologic } \\
\text { factors }\end{array}$} & \multicolumn{3}{|c|}{ Univariate analysis } & \multicolumn{3}{|c|}{ Multivariate analysis } \\
\hline & OR & $95 \%$ CI & $P$ value & OR & $95 \%$ CI & $P$ value \\
\hline \multicolumn{7}{|l|}{ Age (years) } \\
\hline$\leq 67$ & 1 & - & - & & & \\
\hline$>67$ & 1.40 & $0.65-3.00$ & 0.39 & & & \\
\hline \multicolumn{7}{|l|}{ Sex } \\
\hline Female & 1 & - & - & & & \\
\hline Male & 1.19 & $0.54-2.59$ & 0.67 & & & \\
\hline \multicolumn{7}{|l|}{ Ethnicity } \\
\hline Non-Chinese & 1 & - & - & & & \\
\hline Chinese & 3.14 & $0.45-29.5$ & 0.23 & & & \\
\hline \multicolumn{7}{|l|}{ Presence of comorbidities } \\
\hline No & 1 & - & - & & & \\
\hline Yes & 1.21 & $0.42-2.89$ & 0.28 & & & \\
\hline \multicolumn{7}{|l|}{ Macroscopic type } \\
\hline Other types & 1 & - & - & 1 & - & - \\
\hline Diffuse ulcerative and infiltrative & 5.49 & $1.50-20.11$ & $0.01 *$ & 2.69 & $0.65-11.17$ & 0.17 \\
\hline \multicolumn{7}{|l|}{ Histologic type } \\
\hline Differentiated & 1 & - & - & 1 & - & - \\
\hline Poorly differentiated & 2.87 & $0.97-8.55$ & 0.06 & 1.82 & $0.53-6.28$ & 0.34 \\
\hline \multicolumn{7}{|l|}{ Vascular involvement } \\
\hline Negative & 1 & - & - & 1 & - & - \\
\hline Positive & 7.58 & $2.59-22.15$ & $<0.001^{*}$ & 7.37 & $2.33-23.26$ & $0.001 *$ \\
\hline \multicolumn{7}{|l|}{ Lauren's classification } \\
\hline Intestinal or mixed & 1 & - & - & & & \\
\hline Diffuse & 0.76 & $0.32-1.82$ & 0.54 & & & \\
\hline \multicolumn{7}{|l|}{ Depth of invasion (pT) } \\
\hline $\mathrm{T} 3$ & 1 & - & - & 1 & - & - \\
\hline $\mathrm{T} 4$ & 6.77 & $2.43-18.87$ & $<0.001 *$ & 6.56 & $2.11-20.34$ & $0.001 *$ \\
\hline \multicolumn{7}{|l|}{ Nodal involvement } \\
\hline Negative & 1 & - & - & 1 & - & - \\
\hline Positive & 2.68 & $1.05-6.83$ & $0.04 *$ & 0.61 & $0.18-2.15$ & 0.45 \\
\hline \multicolumn{7}{|l|}{ Distant metastasis } \\
\hline Negative & 1 & - & - & 1 & - & - \\
\hline Positive & 5.20 & $2.10-12.86$ & $<0.001 *$ & 4.32 & $0.41-7.29$ & $0.01 *$ \\
\hline
\end{tabular}

* Significant

OR, odds ratio; $95 \%$ CI, $95 \%$ confidence interval

$47.2 \%$ and its negative predictive value, $86.8 \%$. Patients who had advanced macroscopic features, presence of vascular invasion, nodal involvement, increased depth of tumor invasion (pT), and metastatic disease tended to have positive lavage results by univariate analysis. Using multiple logistic regression analysis by a backward stepwise selection procedure, the most significant factors were depth of tumor invasion ( $\mathrm{pT}$ ) and vascular invasion (Table 2).

Among patients who had undergone curative resections $(n=93)$, those with positive peritoneal lavage $(5 / 10)$ had a 15 times higher risk of peritoneal recurrence than those with negative lavage $(5 / 83)$ results (odds ratio [OR], 15.6; 95\% confidence intervals [CI],
2.71-97.67, $P=0.001)$. Further, there was a trend towards earlier recurrences in patients with positive lavage (median, 8 months versus 11 months; $P=0.37$ by $\log$ rank test). Peritoneal dissemination occurred in $42 \%(10 / 24)$ of those with recurrent disease and was the most common clinical presentation in this study population. Recurrence secondary to hematogenous spread was diagnosed in 4 patients, including 2 patients with hepatic, 1 with pulmonary, and 1 with bone metastases. Recurrence at regional lymph nodes was found in 3 patients, while 5 patients had recurrence in the gastric bed or remnant. Disease-free survival was worse for those with positive lavage (median, of 27 months versus 53 months; $P=0.004$ by $\log$ rank test; Fig. 1). By 
Disease-free Survival

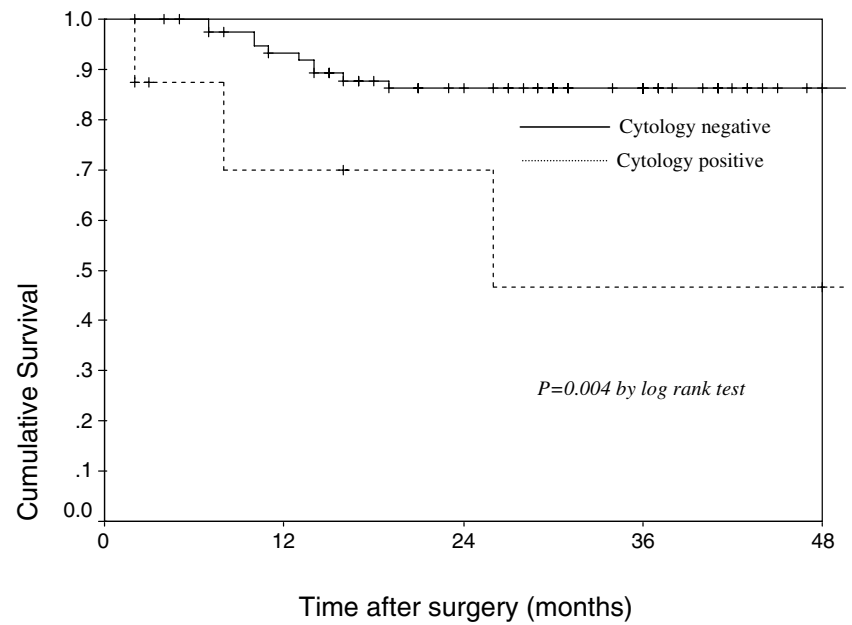

Fig. 1. Disease-free survival for curative resections according to lavage status

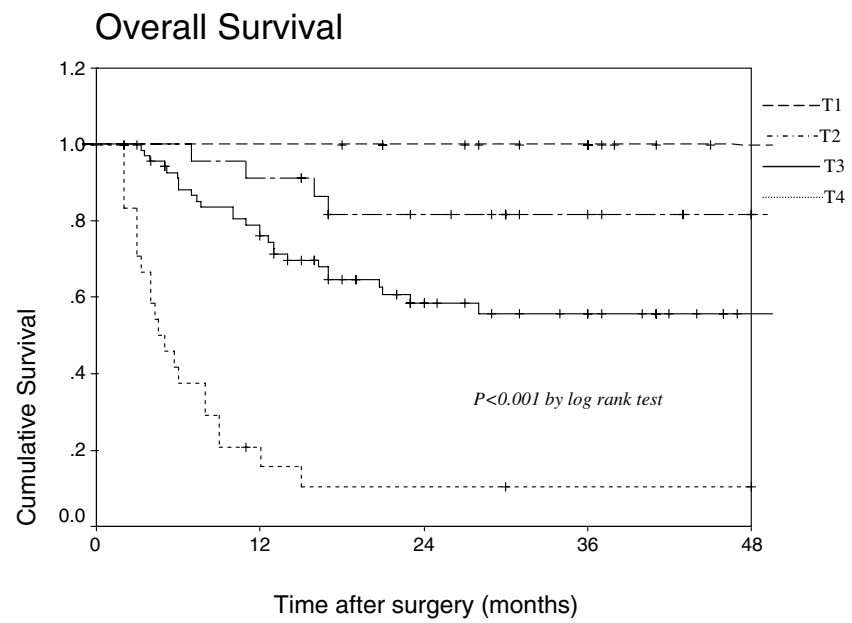

Fig. 2. Overall survival by depth of invasion (pT stage)

multivariate Cox regression analysis by stepwise backward procedure, advanced depth of tumor invasion and positive lavage cytology were independent poor prognostic factors for disease-free survival (Table 3).

Because advanced depth of tumor invasion exerted a significant effect on overall survival (Fig. 2), the group of patients with serosa-positive tumors was analyzed separately. Those with positive cytology had a more, dismal overall survival (Fig. 3), with a median survival of 7 months versus 23 months $(P<0.0001$ by log rank test). Positive lavage cytology remained as a significant factor for poor disease-free survival among those who underwent curative resections (Table 4). Even among patients who had undergone a non-curative procedure, their survival was significantly worse if their lavage cytology had been positive (median survival, 7 months

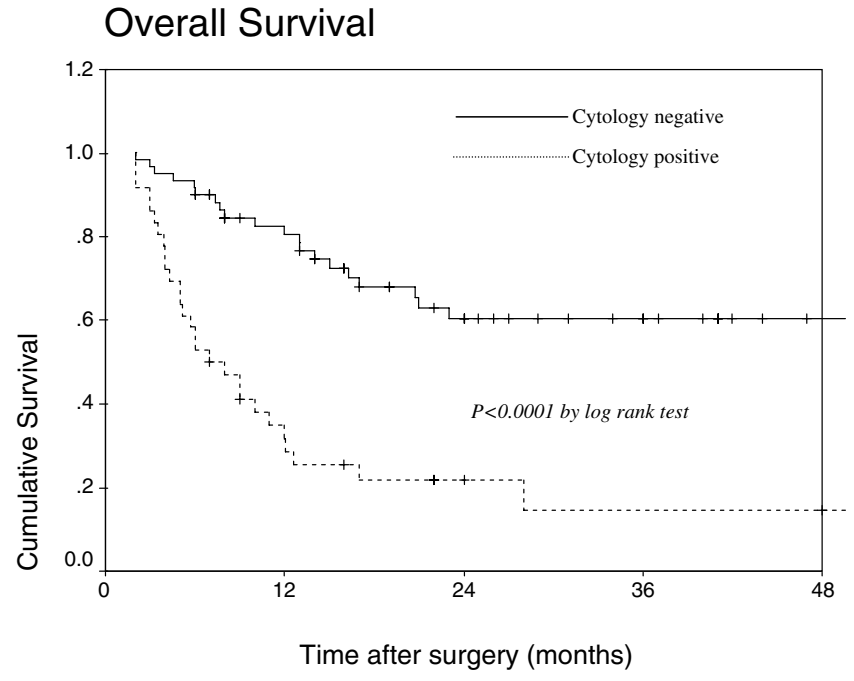

Fig. 3. Overall survival of patients with serosa-positive gastric cancer (pT3, pT4) according to cytology status

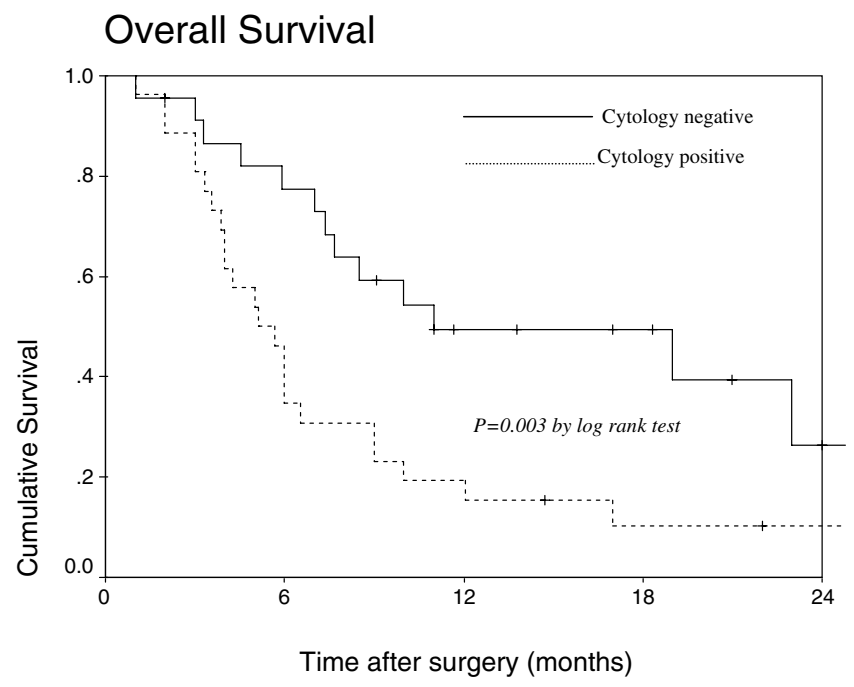

Fig. 4. Overall survival for palliative procedures according to lavage status

versus 14 months for negative lavage cytology $(P=0.003$ by log rank test; Fig. 4).

\section{Discussion}

The prognosis of gastric cancer has altered little despite extensive nodal resection and advances in the adjuvant chemotherapeutic armamentarium. Increased detection of earlier-stage disease, and advances in preoperative staging and perioperative care have improved patient selection and operative survival, translating into improved long-term survival for patients with resectable 
Table 3. Univariate and multivariate Cox regression analysis to predict disease-free survival for patients who underwent curative resections $(n=93)$

\begin{tabular}{|c|c|c|c|c|c|c|}
\hline \multirow{2}{*}{$\begin{array}{l}\text { Clinicopathologic } \\
\text { factors }\end{array}$} & \multicolumn{3}{|c|}{ Univariate analysis } & \multicolumn{3}{|c|}{ Multivariate analysis } \\
\hline & HR & $95 \% \mathrm{CI}$ & $P$ value & HR & $95 \% \mathrm{CI}$ & $P$ value \\
\hline \multicolumn{7}{|l|}{ Age (years) } \\
\hline$\leq 67$ & 1 & - & - & & & \\
\hline$>67$ & 1.24 & $0.46-3.34$ & 0.67 & & & \\
\hline \multicolumn{7}{|l|}{ Sex } \\
\hline Female & 1 & - & - & & & \\
\hline Male & 1.80 & $0.67-4.79$ & 0.24 & & & \\
\hline \multicolumn{7}{|l|}{ Ethnicity } \\
\hline Non-Chinese & 1 & - & - & & & \\
\hline Chinese & 1.88 & $1.24-19.86$ & 0.36 & & & \\
\hline \multicolumn{7}{|l|}{ Presence of comorbidities } \\
\hline No & 1 & - & - & & & \\
\hline Yes & 1.18 & $0.24-3.19$ & 0.54 & & & \\
\hline \multicolumn{7}{|l|}{ Macroscopic type } \\
\hline Other types & 1 & - & - & 1 & - & - \\
\hline Diffuse ulcerative and infiltrative & 4.01 & $1.49-15.20$ & $0.01^{*}$ & 1.15 & $0.25-4.26$ & 0.46 \\
\hline \multicolumn{7}{|l|}{ Histologic type } \\
\hline Differentiated & 1 & - & - & & & \\
\hline Poorly differentiated & 1.16 & $0.38-3.53$ & 0.80 & & & \\
\hline \multicolumn{7}{|l|}{ Vascular involvement } \\
\hline Negative & 1 & - & - & 1 & - & - \\
\hline Positive & 4.20 & $7.38-13.00$ & $0.01 *$ & 1.20 & $0.33-4.36$ & 0.78 \\
\hline \multicolumn{7}{|l|}{ Lauren's classification } \\
\hline Intestinal or mixed & 1 & - & - & & & \\
\hline Diffuse & 1.53 & $0.50-4.68$ & 0.46 & & & \\
\hline \multicolumn{7}{|l|}{ Depth of invasion (pT) } \\
\hline $\mathrm{T} 1, \mathrm{~T} 2$ & 1 & - & - & 1 & - & - \\
\hline $\mathrm{T} 3$ & 7.11 & $1.57-20.52$ & $0.01^{*}$ & 8.98 & $2.52-15.46$ & $0.02 *$ \\
\hline \multicolumn{7}{|l|}{ Nodal involvement } \\
\hline Negative & 1 & - & - & 1 & - & - \\
\hline Positive & 4.77 & $1.31-17.39$ & $0.02 *$ & 4.31 & $1.45-24.32$ & 0.07 \\
\hline \multicolumn{7}{|l|}{ Peritoneal cytology } \\
\hline Negative & 1 & - & - & 1 & - & - \\
\hline Positive & 7.12 & $2.18-18.24$ & $0.01 *$ & 11.51 & $3.05-28.12$ & $<0.001 *$ \\
\hline
\end{tabular}

* Significant

HR, hazard ratio; $95 \%$ CI, 95\% confidence interval

cancers [10]. However, the prognosis for advanced cancer remains dismal, with an overall 5-year survival rate of about $20 \%[11,12]$.

Peritoneal metastasis is the most frequent cause of death in such patients, with an average survival of only 6 months after peritoneal recurrence [13]. In patients with serosal involvement, $50 \%$ developed peritoneal recurrence even if curative resections were performed $[14,15]$. In our series, our findings reflect this trend, with $41 \%(20 / 49)$ of T3 tumors ending up with disease recurrence despite curative resections; out of which half (10/20) recurred as peritoneal disease. Analysis of prognostic factors is therefore important in this group of patients.
Cytological examination of peritoneal lavage fluid has been proposed as a useful predictor of peritoneal recurrence in gastric cancer patients, based on several studies reporting a close correlation between peritoneal carcinomatosis and serosal invasion or the seeding of free cancer cells [16,17]. Iitsuka et al. [18] had observed that the presence of free intraperitoneal cancer cells relied on cancer invasion of the serosa, because cytological positivity did not occur in patients without serosal invasion. Similarly, our study found serosal invasion to be associated with a higher likelihood of cyotological positivity. None of the patients with pT1 and pT2 tumors had positive cytology results. Further, several large series have demonstrated a prognostic role for 
Table 4. Univariate and multivariate Cox regression analysis to predict disease-free survival for patients with serosa-positive tumors who underwent curative resections $(n=45)$

\begin{tabular}{|c|c|c|c|c|c|c|}
\hline \multirow{2}{*}{$\begin{array}{l}\text { Clinicopathologic } \\
\text { factors }\end{array}$} & \multicolumn{3}{|c|}{ Univariate analysis } & \multicolumn{3}{|c|}{ Multivariate analysis } \\
\hline & HR & $95 \% \mathrm{CI}$ & $P$ value & HR & $95 \% \mathrm{CI}$ & $P$ value \\
\hline \multicolumn{7}{|l|}{ Age (years) } \\
\hline$\leq 67$ & 1 & - & - & & & \\
\hline$>67$ & 1.15 & $0.38-3.42$ & 0.81 & & & \\
\hline \multicolumn{7}{|l|}{ Sex } \\
\hline Female & 1 & - & - & & & \\
\hline Male & 0.19 & $0.14-1.14$ & 0.39 & & & \\
\hline \multicolumn{7}{|l|}{ Ethnicity } \\
\hline Non-Chinese & 1 & - & - & & & \\
\hline Chinese & 1.92 & $0.74-4.23$ & 0.41 & & & \\
\hline \multicolumn{7}{|l|}{ Presence of comorbidities } \\
\hline No & 1 & - & - & & & \\
\hline Yes & 1.02 & $0.12-2.21$ & 0.76 & & & \\
\hline \multicolumn{7}{|l|}{ Macroscopic type } \\
\hline Other types & 1 & - & - & 1 & - & - \\
\hline Diffuse ulcerative and infiltrative & 6.41 & $1.51-17.20$ & $0.01 *$ & 2.33 & $1.01-5.40$ & 0.08 \\
\hline \multicolumn{7}{|l|}{ Histologic type } \\
\hline Differentiated & 1 & - & - & & & \\
\hline Poorly differentiated & 0.86 & $0.37-1.97$ & 0.72 & & & \\
\hline \multicolumn{7}{|l|}{ Vascular involvement } \\
\hline Negative & 1 & - & - & 1 & - & - \\
\hline Positive & 1.87 & $0.84-4.14$ & 0.12 & 1.04 & $0.40-2.69$ & 0.93 \\
\hline \multicolumn{7}{|l|}{ Lauren's classification } \\
\hline Intestinal or mixed & 1 & - & - & & & \\
\hline Diffuse & 1.01 & $0.46-2.20$ & 0.98 & & & \\
\hline \multicolumn{7}{|l|}{ Nodal involvement } \\
\hline Negative & 1 & - & - & 1 & - & - \\
\hline Positive & 1.95 & $0.86-4.41$ & 0.11 & 1.21 & $0.56-3.54$ & 0.21 \\
\hline \multicolumn{7}{|l|}{ Peritoneal cytology } \\
\hline Negative & 1 & - & - & 1 & - & - \\
\hline Positive & 8.06 & $1.33-15.56$ & $0.02 *$ & 4.23 & $1.24-12.63$ & $0.01 *$ \\
\hline
\end{tabular}

* Significant

HR, hazard ratio; 95\% CI, 95\% confidence interval

cytological findings $[1,10,15,16]$. Nakajima et al. [10] found that survival rates were significantly lower when cytological examination was positive - even when patients were in the same clinical stage. Bando et al. [1] proposed peritoneal lavage cytological examination as a useful predictor of peritoneal recurrence after curative resections, with a sensitivity of $56 \%$ and specificity of $97 \%$. Their series found a $30 \%$ incidence of positive malignant cytology among serosal-invasive tumors and a $49 \%$ incidence among those with gross peritoneal disease. Positive cytology was an independent poor prognostic factor for overall survival, with 5-year survival rates of $2 \%$ and $58 \%$ for those with positive and negative cytology, respectively. Though the study population in our series was small, close parallels can be observed - we found a $26.4 \%$ incidence of positive malignant cytology among serosa-positive tumors and an inci- dence of $70.8 \%$ among those with gross peritoneal disease. Positive cytological examination, advanced serosal invasion, and metastatic disease, including peritoneal dissemination, were independent prognostic indicators for poor overall survival (Table 5). Multivariate analysis, using the Cox stepwise hazard model, revealed that the cytological findings were statistically significant for disease-free survival among the other prognostic factors selected by univariate analysis (Table 3 ). This remained true even after controlling for $\mathrm{T}$ stage (Table 4). These results suggest that peritoneal cytological findings have a strong independent prognostic influence following curative resection. Even for those who underwent palliative procedures, survival was significantly worse for those with positive cytology results (mean, 7 months versus 14 months; $P=0.003$ by log rank test). We also observed a trend of earlier recurrences in the group 
E.W.L. Chuwa et al.: Peritoneal lavage cytology for gastric cancer in Singapore

Table 5. Univariate and multivariate Cox regression analysis to predict overall survival for patients with serosa-positive (pT3, pT4) tumors $(n=96)$

\begin{tabular}{|c|c|c|c|c|c|c|}
\hline \multirow{2}{*}{$\begin{array}{l}\text { Clinicopathologic } \\
\text { factors }\end{array}$} & \multicolumn{3}{|c|}{ Univariate analysis } & \multicolumn{3}{|c|}{ Multivariate analysis } \\
\hline & HR & $95 \%$ CI & $P$ value & HR & $95 \% \mathrm{CI}$ & $P$ value \\
\hline \multicolumn{7}{|l|}{ Age (years) } \\
\hline$\leq 67$ & 1 & - & - & & & \\
\hline$>67$ & 0.71 & $0.42-1.17$ & 0.18 & & & \\
\hline \multicolumn{7}{|l|}{ Sex } \\
\hline Female & 1 & - & - & & & \\
\hline Male & 1.19 & $0.70-2.01$ & 0.52 & & & \\
\hline \multicolumn{7}{|l|}{ Ethnicity } \\
\hline Non-Chinese & 1 & - & - & & & \\
\hline Chinese & 2.04 & $0.67-11.22$ & 0.20 & & & \\
\hline \multicolumn{7}{|l|}{ Presence of comorbidities } \\
\hline No & 1 & - & - & & & \\
\hline Yes & 1.42 & $0.82-3.02$ & 0.62 & & & \\
\hline \multicolumn{7}{|l|}{ Macroscopic type } \\
\hline Other types & 1 & - & - & 1 & - & - \\
\hline Diffuse ulcerative and infiltrative & 3.50 & $1.38-8.86$ & $0.008 *$ & 1.51 & $0.55-4.14$ & 0.43 \\
\hline \multicolumn{7}{|l|}{ Histologic type } \\
\hline Differentiated & 1 & - & - & & & \\
\hline Poorly differentiated & 0.99 & $0.52-1.92$ & 0.99 & & & \\
\hline \multicolumn{7}{|l|}{ Vascular involvement } \\
\hline Negative & 1 & - & - & 1 & - & - \\
\hline Positive & 1.77 & $0.96-3.27$ & 0.07 & 0.91 & $0.42-1.94$ & 0.80 \\
\hline \multicolumn{7}{|l|}{ Lauren's classification } \\
\hline Intestinal or mixed & 1 & - & - & 1 & - & - \\
\hline Diffuse & 0.60 & $0.32-1.12$ & 0.11 & 1.24 & $0.56-2.75$ & 0.59 \\
\hline \multicolumn{7}{|l|}{ Depth of invasion (pT) } \\
\hline T3 & 1 & - & - & 1 & - & - \\
\hline $\mathrm{T} 4$ & 5.51 & $3.04-9.98$ & $<0.001 *$ & 2.21 & $1.11-4.41$ & $0.02 *$ \\
\hline \multicolumn{7}{|l|}{ Nodal involvement } \\
\hline Negative & 1 & - & - & 1 & - & - \\
\hline Positive & 2.30 & $1.19-4.44$ & $0.01 *$ & 1.06 & $0.48-2.33$ & 0.89 \\
\hline \multicolumn{7}{|l|}{ Distant metastasis } \\
\hline Negative & 1 & - & - & 1 & - & - \\
\hline Positive & 5.32 & $3.23-11.52$ & $<0.001 *$ & 3.89 & $2.62-10.35$ & $0.01 *$ \\
\hline \multicolumn{7}{|l|}{ Peritoneal cytology } \\
\hline Negative & 1 & - & - & 1 & - & - \\
\hline Positive & 4.35 & $2.42-7.81$ & $<0.001 *$ & 2.31 & $1.19-4.47$ & $0.01 *$ \\
\hline
\end{tabular}

* Significant

HR, hazard ratio; $95 \%$ CI, $95 \%$ confidence interval

with positive cytology results (median, 8 months versus 11 months; $P=0.37$ ). This trend-failed to reach statistical significance, although this could be explained by the small sample size and perhaps an inadequate follow-up period. Moreover, analysis of recurrence patterns varies widely - with inconsistencies related to different surgical treatments, as well as the mode and timing of detection of recurrence.

Our study also revealed that tumors with vascular invasion had a significantly higher rate of positive cytological findings than those without (Table 2). These re- sults suggest that such tumors may have a propensity for peritoneal dissemination. This adds to the existing theories of peritoneal carcinomatosis that suggest the shedding of cancer cells from the serosal surface of the primary tumor [17] and metastatic routes via the lymphatic system [19].

In the West, peritoneal lavage for cytological examination is not routine. The Dutch Gastric Cancer Trial demonstrated positive cytology in only $7.1 \%$ of all patients with gastric cancer and in $12 \%$ of those with serosal invasion [20]. Though the incidence of positive 
cytology was lower in their series than that in Japanese studies, a $10.65 \%(57 / 535)$ incidence of missing or inconclusive cytology results probably implied inconsistent handling and interpretation of results, hence reducing the detection rate. Nonetheless, they demonstrated a significant association of positive cytology results with serosal invasion and lymph node infiltration, and they showed that the survival of patients with positive cytology results was significantly worse than that of patients with negative findings. Hayes et al. [21] from the Northern Oesophagogastric Cancer Unit in the United Kingdom, found a 27\% incidence of positive peritoneal malignant cytology. In their study, they had gone further to demonstrate that the yield of free cells could be significantly increased by the addition of both brush cytology and imprint cytology. Again, survival was significantly worse in the group with positive cytology, with a survival of $22 \%$ at 2-year follow-up compared to $66 \%$ for the cytology-negative group $(P<0.0001)$.

Despite these encouraging reports, the overall accuracy of cytological examination has been criticized as being variable; ranging from $22 \%$ to $30 \%$ in gastric carcinoma involving the serosa [6-8]. This can be explained by the non-standardized methods of performimg peritoneal lavage and the presence of interobserver bias between cytopathologists in grading the cytological findings. Abe et al. [7] pointed out that false-negative cases might arise from technical flaws, such as incomplete sampling, that were not representative of the lavage process. In patients with early peritoneal dissemination, cancer cells may reside deep in the mesothelial cell layer and, hence, would not be exposed to the peritoneal surface.

More sensitive methods for the early detection of peritoneal dissemination were deemed necessary. This brought about various methods aimed either at refining the cell-yield technique through molecular approaches or targeting a different factor altogether.

Nakanishi et al. [22] demonstrated that carcinoembryonic antigen (CEA) concentrations in peritoneal washings were independent of those in paired serum samples and could predict peritoneal metastasis more reliably than conventional cytological study. Kodera et al. [23] followed this with the use of reverse transcriptase-polymerase chain reaction (RT-PCR) analysis with primers specific for CEA to improve the sensitivity of peritoneal lavage analysis. Based on investigations by Ohta et al. [24] showing that human gastric cancers immunoreacted with a monoclonal antibody against pancreatic trypsinogen, Fujimura et al. [25] found that trypsinogen protein and trypsinogen- 1 mRNA expression demonstrated by RT-PCR could serve as a novel marker to detect early peritoneal dissemination of gastric cancer.
These novel methods suffer from being tedious to perform, time-consuming, and costly; as well, they require specialized laboratory facilities and trained expertise to carry out. More importantly, definitive results for diagnosis could not be reached at the time of the operation. Further technical refinements have been suggested to achieve rapid analysis [26,27]. Having a diagnostic result while the operation is still in progress could help guide surgical and adjuvant therapy.

Though it has long been appreciated that patients with advanced gastric cancer offered surgery alone have little hope of being cured, the past decade has witnessed renewed interest in adjuvant therapeutic approaches aimed at improving the survival of these patients. A recently published meta-analysis of 21 randomized studies on the value of adjuvant treatment for gastric cancer revealed a survival benefit for treated patients [28]. The recently concluded findings of the United Kingdom MAGIC trial [29] marked the first large randomized trial to support the efficacy of perioperative chemotherapy for advanced gastric cancer. Progressionfree survival was significantly prolonged (hazard ratio [HR], 0.70; $95 \mathrm{CI}, 0.56-0.88 ; P=0.002$ ) in the group randomized to chemotherapy. However, comparison of overall survival at 2 years between the group who received perioperative chemotherapy $(48 \%)$ and the group who underwent surgery alone $(40 \%)$ showed no statistically significant difference. The trial has been criticized both for lacking routine standardized preoperative staging assessments and for lacking surgical quality control concerning the extent of lymphadenectomy.

If we recognize peritoneal recurrence as the chief cause of surgical treatment failure and mortality after curative resections, then intraperitoneal chemotherapy is a viable modality to eradicate microscopic residual disease, or as a prophylactic measure. However, like systemic chemotherapy, this too remains controversial. Although randomized trials of adjuvant perioperative intraperitoneal chemotherapy have demonstrated significant improvement in survival compared with surgery alone [5,30], this finding was not supported by multicenter phase III studies [31; Miyashiro et al., unpublished data]. Other modalities that have been explored include intraperitoneal administration of carbon-adsorbed mitomycin C [32] or OK432 [33] and cytoreductive surgery, together with systemic intraoperative hyperthermochemotherapy [34].

Adjuvant chemotherapy, whether systemic or intraperitoneal, is costly, with no added benefit to patients without residual disease; and it is not without menacing toxicity and morbidity. Detection of free cancer cells can therefore be of great value in the selection of patients who may benefit from further aggressive therapy. Such defection helps to select candidates 
for neoadjuvant and adjuvant chemotherapy trials and avoids unnecessary laparotomies in patients in whom only palliation is appropriate. A prospective multicenter study in Europe - Evolution of Peritoneal Carcinomatosis (EVOCAPE) 2 - is currently investigating the prognostic value of peritoneal cytological examination for predicting peritoneal carcinomatosis. The results would better define a patient group for whom adjuvant chemotherapy would be indicated. Setting our findings in perspective, this simple procedure should be included in our current management of our patients with gastric cancer.

\section{References}

1. Bando E, Yonemura Y, Takeshita Y, et al. Intraoperative lavage for cytological examination in 1297 patients with gastric carcinoma. Am J Surg 1999;178:256-62.

2. Wu CC, Chen JT, Chang MC, et al. Optimal surgical strategy for potentially curative serosa-involved gastric carcinoma with intraperitoneal free cancer cells. J Am Coll Surg 1997;184:611-7.

3. Japanese Research Society for Gastric Cancer. Japanese classification of gastric carcinoma. 2nd English ed. Gastric Cancer 1998;1:11-24.

4. Ajani JA, Mansfield PF, Janjan N, et al. Multiinstitutional trial of preoperative chemoradiotherapy in patients with potentially resectable gastric carcinoma. J Clin Oncol 2004;22:2774-80.

5. Kim JY, Bae HS. A controlled clinical study of serosa-invasive gastric carcinoma patients who underwent surgery plus intraperitoneal hyperthermo-chemo-perfusion (IHCP). Gastric Cancer 2001;4:27-33.

6. Suzuki T, Ochiai T, Hayashi, H, et al. Peritoneal lavage cytology findings as prognostic factor for gastric cancer. Semin Surg Oncol 1999;17:103-7.

7. Abe S, Yoshimura H, Tabata $\mathrm{H}$, et al. Curative resection of gastric cancer: limitation of peritoneal lavage cytology in predicting the outcome. J Surg Oncol 1995;59:226-9.

8. Boku T, Nakane Y, Minoura T, et al. Prognostic significance of serosal invasion and free intraperitoneal cancer cells in gastric cancer. Br J Surg 1990;77:436-9.

9. Papanicolau GN. Atlas of exfoliative cytology. Cambridge: Harvard University Press; 1963.

10. Nakajima T, Nishi M, Kajitani T. Improvement in treatment results of gastric cancer with surgery and chemotherapy: experience of 9700 cases in the Cancer Institute Hospital, Tokyo. Semin Surg Oncol 1991;7:365-72.

11. Fuchs CS, Mayer RJ. Gastric carcinoma. N Engl J Med 1995; 333:32-41.

12. Hundahl SA, Menck HR, Mansour EG. The National Cancer Database report on gastric carcinoma. Cancer 1997;80:2333-41.

13. Maekawa S, Saku M, Maehara Y, et al. Surgical treatment for advanced gastric cancer. Hepatogastroenterology 1996;43:17886.

14. Moriguchi S, Maehara Y, Korenaga D, et al. Risk factors which predict pattern of recurrence after curative surgery for patients with advanced gastric cancer. Surg Oncol 1992;1:341-6.

15. Iitsuka Y, Shiota S, Matsui T, et al. Relationship between the cytological characteristics of intraperitoneal free cancer cells and the prognosis in patients with gastric cancer. Acta Cytol 1990;34: 437-42.
16. Ikeguchi M, Oka A, Tsujitani S, et al. Relationship between area of serosal invasion and intraperitoneal free cancer cells in patients with gastric cancer. Anticancer Res 1994;14:2131-4.

17. Yonemura Y, Nojima N, Kawamura T, et al. Mechanisms of the formation of peritoneal dissemination. In: Yonemura Y, editor. Peritoneal dissemination. Molecular mechanisms and the latest therapy. Kanazawa, Japan: Maeda Shoten;1998:1-46.

18. Iitsuka Y, Kaneshima S, Tanida O, et al. Intraperitoneal free cancer cells and their viability in gastric cancer. Cancer 1979;44: 1476-80.

19. Sampson JA. Implantation peritoneal carcinomatosis of ovarian origin. Am J Pathol 1931;7:423-43.

20. Bonenkamp JJ, Songun I, Hermans J, et al. Prognostic value of positive cytology findings from abdominal washings in patients with gastric cancer. Br J Surg 1996;83:672-4.

21. Hayes N, Wayman J, Wadehra V, et al. Peritoneal cytology in the surgical evaluation of gastric carcinoma. Br J Cancer 1999;79:5204.

22. Nakanishi H, Kodera Y, Torii A, et al. Detection of carcinoembryonic antigen-expressing free tumor cells in peritoneal washes from patients with gastric cancer. Jpn J Cancer Res 1997;88:687-92.

23. Kodera Y, Nakanishi H, Yamamura Y, et al. Prognostic value and clinical implications of disseminated cancer cells in the peritoneal cavity detected by reverse transcriptase-polymerase chain reaction and cytology. Int J Cancer 1998;79:429-33.

24. Ohta T, Terada T, Nagakawa T, et al. Expression of pancreatic trypsinogen in human extrapancreatic gastrointestinal carcinomas. Oncol Rep 1994;1:759-64.

25. Fujimura $\mathrm{T}$, Ohta $\mathrm{T}$, Kitagawa $\mathrm{H}$, et al. Trypsinogen expression and early detection for peritoneal dissemination in gastric cancer. J Surg Oncol 1998;69:71-5.

26. Nakanishi H, Kodera Y, Yamamura Y, et al. Rapid quantitative detection of carcinoembryonic antigen-expressing free tumour cells in the peritoneal cavity of gastric cancer patients with realtime RT-PCR on the LightCycler. Int J Cancer 2000;89:411-7.

27. Nomoto S, Nakao A, Takeuchi Y, et al. Intraoperative peritoneal washing cytology with the rapid immunoperoxidase method using microwave irradiation. J Surg Oncol 1995;60:30-4.

28. Earle CC, Maroun JA. Adjuvant chemotherapy after curative resection for gastric cancer in non-Asian patients; revisiting a meta-anlysis of randomized trials. Eur J Cancer 1999;35:1059-64.

29. Cunningham D, Allum W, Weeden S. Perioperative chemotherapy in operable gastric and lower oesophageal cancer. A randomised contolled trial of the UK Upper GI Clinical Studies Group (the MAGIC trial ISRCTN 93793971). Eur J Cancer Suppl; 2003;1:S18.

30. Yonemura Y, de Arexabala X, FujimuraT, et al. Intraoperative chemohyperthermic peritoneal perfusion as an adjuvant to gastric cancer: final results of a randomized controlled study. Hepatogastroenterology 2001;48:1776-82.

31. Sautner T, Hofbauer F, Depisch D, et al. Adjuvant intraperitoneal cisplatin chemotherapy does not improve long-term survival after surgery for advanced gastric cancer. J Clin Oncol 1994;12: 970-4.

32. Hagiwara A, Takahashi T, Kojima O, et al. Prophylaxis with carbon-adsorbed mitomycin against peritoneal recurrences of gastric cancer. Lancet 1992;339:629-31.

33. Torisu M, Katano M, Kimura Y, et al. New approach to management of malignant ascitis with a streptococcal preparation OK432: improvement of host immunity and prolongation of survival. Surgery 1983;93:353-7.

34. Yonemura Y, Fujimura T, Fushida S, et al. Hyperthermochemotherapy combined with cytoreductive surgery for the treatment of gastric cancer with peritoneal dissemination. World J Surg 1991; 15:530-6. 